\title{
Magnetically arrrested disk around a black hole, and jet formation
}

\section{G. S. Bisnovatyi-Kogan*}

Space Research Institute, Russian Academy of Sciences,

Profsoyuznaya 84/32, Moscow 117997, Russia

and

National Research Nuclear University MEPhI

(Moscow Engineering Physics Institute), Kashirskoe Shosse 31, Moscow 115409, Russia

and

Moscow Institute of Physics and Technology MIPT, Dolgoprudny, Moscow reg., Russia

E-mail: gkogandiki.rssi.ru

The exact time-dependent solution is obtained for a magnetic field growth during a spherically symmetric accretion into a black hole $(\mathrm{BH})$ with a Schwarzschild metric. Magnetic field is increasing with time, changing from the initially uniform into a quasi-radial field. Equipartition between magnetic and kinetic energies in the falling gas is supposed to be established in the developed stages of the flow. Estimates of the synchrotron radiation intensity are presented for the stationary flow. The two-dimensional stationary self-similar magnetohydrodynamic solution is obtained for the matter accretion into $\mathrm{BH}$, in a presence of a large-scale magnetic field, under assumption, that the magnetic field far from the $\mathrm{BH}$ is homogeneous. At the symmetry plane perpendicular to the direction of the distant magnetic field, the dense quasi-stationary disk is formed around $\mathrm{BH}$, which structure is determined by dissipation processes. The radiative efficiency of the magnetized disk is very high, reaching $\sim 0.5 \dot{M} c^{2}$. This model of accretion was called recently as a magnetically arrested disk (MAD). Numerical simulations of MAD, and its appearance during accretion into neutron stars are considered and discussed.

High Energy Phenomena in Relativistic Outflows VII - HEPRO VII

9-12 July 2019

Facultat de Física, Universitat de Barcelona, Spain

\footnotetext{
* Speaker.
} 


\section{Magnetic field evolution in spherical accretion}

Consider changes in the frozen magnetic field structure, without a back reaction, in the spherical accretion flow onto a black hole with a mass $M$, in presence of a free-fall velocity, and initially uniform magnetic field. It is described by following equations, written in GR [G]

$$
\frac{d}{d t}\left(\sqrt{-g} u_{0}^{-1} B^{r}\right)=0 ; \quad \frac{d}{d t}\left(\sqrt{-g} u_{0}^{-1} B^{\theta}\right)=0 ; \quad \frac{d}{d t}=\frac{\partial}{\partial t}+\frac{u^{r}}{u^{0}} \frac{\partial}{\partial r} .
$$

The first and the second equations in $(\mathbb{L} \mathbb{C})$ are related to the conservation of the magnetic flux along the radial and tangential directions. In Newtonian limit the following solution for the evolution with time of the $r$ and $\theta$ physical components of the magnetic field was obtained [ $[$ ] , see also [ $[$ ] .

$$
\begin{gathered}
r B=\left(1+\frac{3}{2} \frac{c t \sqrt{r_{g}}}{r^{3 / 2}}\right)^{4 / 3} B_{0} \cos \theta, \quad{ }_{\theta} B=-\left(1+\frac{3}{2} \frac{c t \sqrt{r_{g}}}{r^{3 / 2}}\right)^{1 / 3} B_{0} \sin \theta . \\
v_{r}=\alpha c \sqrt{\frac{r_{g}}{r}}, \quad \rho=\frac{1}{\alpha} \frac{\dot{M}}{4 \pi c r^{2}} \sqrt{\frac{r}{r_{g}}}, \quad\left(B_{r}^{2}\right)_{\max }=\rho v_{r}^{2}=\alpha \frac{\dot{M} c}{r^{2}} \sqrt{\frac{r_{g}}{r}}, \quad \alpha \lesssim 1 .
\end{gathered}
$$
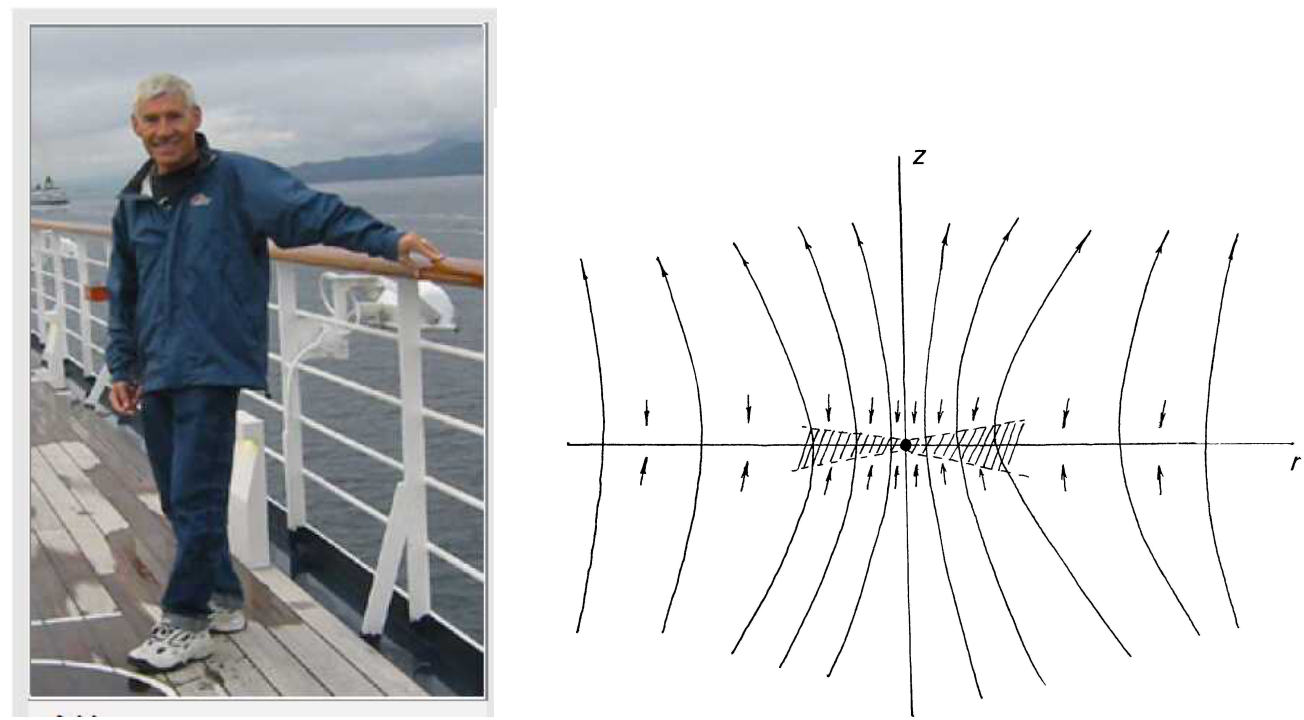

Figure 1: Alexandr Ruzmaikin (Left) A qualitative picture of a stationary accretion of matter with a large scale magnetic field onto BH, Arrows indicate the direction of motion of the matter. The magnetic field far from the star is in the direction of the $z$-axis. The infalling matter forms a disk in the plane $\theta=\pi / 2$, which slowly settles to the star. In the flow region $E_{B} \sim E_{k i n}$, and rotation is entirely absent (Right), from [四].

Sketch of the magnetic field threading an accretion disk is shown in Fig.W. Shown increase of the field owing to flux freezing in the accretion disk matter.

\section{Radiation flux in presence of chaotic magnetic field}

For given values of $\rho_{\infty}, T_{\infty}, M$, the mass flux is determined by 


$$
\dot{M}=4 \pi \rho v_{r} r^{2}=\frac{10^{32}}{c^{2}}\left(\frac{M}{M_{\odot}}\right)^{2}\left(\frac{\rho_{\infty}}{10^{-24} \mathrm{~g} \mathrm{~cm}^{-3}}\right) \times\left(\frac{T_{\infty}}{10^{4} \mathrm{~K}}\right)^{-3 / 2} \mathrm{~g} \mathrm{~s}^{-1} .
$$

The free-free and free-bound radiation of non-relativistic (NR), and ultra-relativistic (UR) maxwellian plasma is determined by formulae [ $[\mathbf{D}]$

$$
\varepsilon_{f f}+\varepsilon_{f b} \approx 2 \times 10^{22} \rho T^{1 / 2} \operatorname{erg~g}^{-1} \mathrm{~s}^{-1}(N R) ; \quad \varepsilon_{f f}^{\mathrm{rel}} \approx 2 \times 10^{16} \rho T \ln \frac{k T}{m_{\mathrm{e}} c^{2}} \operatorname{erg~g}^{-1} \mathrm{~s}^{-1}(U R)
$$

The total luminosity in $L_{t}$ in this case corresponds to efficiency $\varepsilon \sim 10^{-8}$ for average interstellar parameters [ए]3]

$$
L_{t}=L_{f f}+L_{f b}=4 \pi \int_{r_{i n}}^{\infty} \rho\left(\varepsilon_{f f}+\varepsilon_{f b}\right) r^{2} d r \mathrm{erg} \mathrm{s}^{-1}, \quad r_{i n} \approx 1.5 r_{g} .
$$

It was shown in [[13], that in presence of a magnetic field frozen in plasma, the efficiency of the kinetic energy conversion into heat increases drastically, approaching $\eta \approx 10 \%$. When the gas flows radially, the lines of magnetic force stretch along a radius, $B_{r} \sim r^{-2}$, and the magnetic energy per unit volume $E_{M} \sim B^{2} \sim r^{-4}$ increases more rapidly than the kinetic energy $E_{\text {kin }} \sim \rho v^{2} \sim$ $\dot{M} v / r^{2} \sim r^{-5 / 2}\left(\dot{M}=4 \pi \rho v r^{2}\right.$ is the stationary mass flux, free-fall velocity $\left.v \sim r^{-1 / 2}\right)$. Since the energy $E_{M}$ cannot physically exceed $E_{\text {kin }}$, it was assumed in [ए3] that an equipartition of energy $E_{M} \approx E_{\mathrm{kin}}$ is supported by the dissipation of magnetic energy. The excess of magnetic energy is consumed by plasma heating, which was taken into account in [6]. It leads to an increase in efficiency $\eta$ to $30 \%$ that may be considered as a realistic estimate under these assumptions.

If $E_{M} \sim r^{-4}$ is the magnetic field energy with no dissipation, and $E_{M}^{\prime}=E_{\text {kin }} \sim r^{-5 / 2}$ is the energy of the magnetic field in a flow, then an increase of entropy per unit volume along a radius due to the field annihilation in a stationary flow is given by

$$
Q_{M}=\left(\rho T \frac{d S}{d r}\right)_{M}=\left(\frac{d E_{M}}{d r}-\frac{d E_{M}^{\prime}}{d r}\right)_{E_{M}=E_{M}^{\prime}}=-4 \frac{E_{M}}{r}+\frac{5}{2} \frac{E_{M}}{r}=-\frac{3}{2 r} \frac{B^{2}}{8 \pi} .
$$

The energy balance equation

$$
T \frac{d S}{d r}=\frac{d E}{d r}-\frac{P}{\rho^{2}} \frac{d \rho}{d r}=\frac{Q_{M}}{\rho}-\frac{\varepsilon_{B}}{v_{r}}
$$

was solved using (2.4), and the following relations, where $\varepsilon_{B}\left(\mathrm{erg} \mathrm{g}^{-1} \mathrm{~s}^{-1}\right)$ is a rate of magnetobremsstrahlung losses of the Maxwell plasma [ [⿴囗十, 团], with magnetic field equipartition, so that

$$
\begin{gathered}
\frac{B^{2}}{8 \pi}=\frac{1}{2} \rho v_{r}^{2}, \quad v_{r}=\alpha v_{f f}=\alpha \sqrt{\frac{2 G M}{r}, \quad \overline{B_{\perp}^{2}}=\frac{2}{3} \overline{B^{2}},} \\
\varepsilon_{B}=2 \frac{\mathrm{e}^{2}}{m_{\mathrm{p} c}}\left(\frac{e B_{\perp}}{m_{e} c^{2}}\right)^{2} \frac{k T}{m_{e} c^{2}} \approx 0.46 T B_{\perp}^{2} \mathrm{erg} \mathrm{g}^{-1} \mathrm{~s}^{-1} \text { for } k T \ll m_{e} c^{2} \quad(\mathrm{NR}), \\
\varepsilon_{B}=8 \frac{\mathrm{e}^{2}}{m_{\mathrm{p}} c}\left(\frac{\mathrm{e} B_{\perp}}{m_{e} c^{2}}\right)^{2}\left(\frac{k T}{m_{e} c^{2}}\right)^{2} \approx 3.2 \times 10^{-10} T^{2} B_{\perp}^{2} \mathrm{erg} \mathrm{g}^{-1} \mathrm{~s}^{-1} \quad \text { for } k T \gg m_{e} c^{2}
\end{gathered}
$$

As was shown in the paper [5], the condition $T_{e} \approx T_{p}$ is supported in the quasi-spherical accretion flow in presence of a chaotic magnetic field annihilation. It was obtained [ 6 ], that $\varepsilon \sim 0.1$ from [[1]] grows until $\varepsilon \sim 0.3$ with account of heating at magnetic field annihilation. 


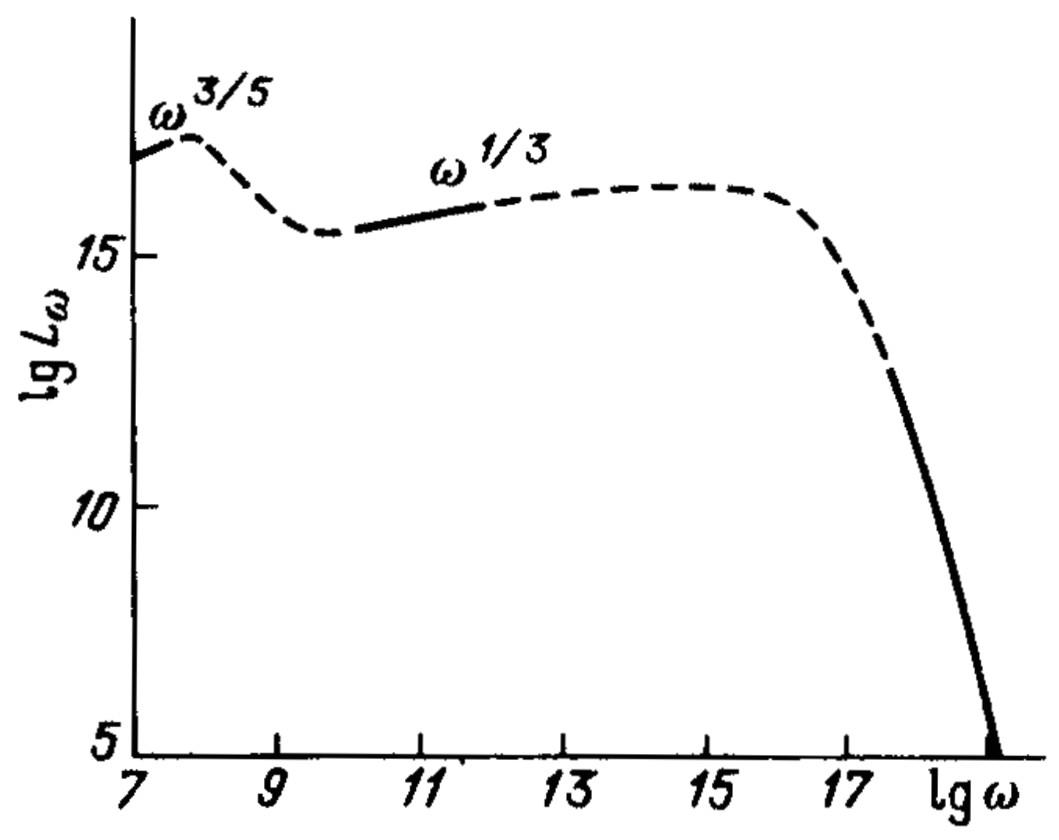

Figure 2: Magneto-bremsstrahlung spectrum of a $\mathrm{BH}$ of $M=10 M_{\odot}$ for a spherically symmetric accretion and random magnetic field at $\rho_{\infty}=10^{-24} \mathrm{~g} \cdot \mathrm{cm}^{-3}, T_{\infty}=10^{4} \mathrm{~K}, \alpha^{2}=1 / 3$. The solid lines represent asymptotic dependencies, dashed lines give extrapolations, from [四].

\section{Self-similar solution for the stationary flow outside the symmetry plane, in presence of a uniform magnetic field at infinity}

Let us consider a flow in the inner supersonic region, where pressure is negligible and such a flow is essentially directed by the magnetic field. In the axial symmetry case, the stationary picture will be two-dimensional. The basic equations for two-dimensional accretion onto a gravitating centre $(\mathrm{BH})$ with mass $M$, of the magnetized gas with perfect conductivity, and without pressure are written in the form [प]

$$
\begin{gathered}
v_{r} \frac{\partial v_{r}}{\partial r}+\frac{v_{\theta}}{r} \frac{\partial v_{r}}{\partial \theta}-\frac{v_{\theta}^{2}}{r}=-\frac{G M}{r^{2}}-\frac{B_{\theta}}{4 \pi \rho r}\left[\frac{\partial\left(r B_{\theta}\right)}{\partial r}-\frac{\partial B_{r}}{\partial \theta}\right], \\
v_{r} \frac{\partial v_{\theta}}{\partial r}+\frac{v_{\theta}}{r} \frac{\partial v_{\theta}}{\partial \theta}+\frac{v_{r} v_{\theta}}{r}=\frac{B_{r}}{4 \pi \rho r}\left[\frac{\partial\left(r B_{\theta}\right)}{\partial r}-\frac{\partial B_{r}}{\partial \theta}\right], \\
\frac{1}{r} \frac{\partial}{\partial r}\left(r^{2} \rho v_{r}\right)+\frac{1}{\sin \theta} \frac{\partial}{\partial \theta}\left(\sin \theta \rho v_{\theta}\right)=0, \\
\frac{1}{r} \frac{\partial}{\partial r}\left(r^{2} B_{r}\right)+\frac{1}{\sin \theta} \frac{\partial}{\partial \theta}\left(\sin \theta B_{\theta}\right)=0, \\
v_{r} B_{\theta}-v_{\theta} B_{r}=0 .
\end{gathered}
$$

We assumed that a picture of the flow is stationary $(d / d t=0)$, two-dimensional $\partial / \partial \phi=0$, and the matter falling to a BH has no angular momentum $v_{\phi}=B_{\phi}=0$. Eqs. (B.D)-(B.2) are the $r$, and $\theta$ components of the Euler equation; (B.3) is the continuity equation; (B.4) defines zero divergency 
of the magnetic field ( $\operatorname{div} \mathbf{B}=0$ ), and (B.5) defines the 'freezing' of the magnetic field, when the flow patterns are parallel to the magnetic field lines. The magnetic force on the right sides of Eqs. (B. ل1),(B.2) is perpendicular to the streamlines. The Bernoulli equation is obtained by multiplying (B.]) by $v_{r}$, (B.2) by $v_{\theta}$ and combining them,

$$
v_{r} \frac{\partial v^{2}}{\partial r}+\frac{v_{\theta}}{r} \frac{\partial v^{2}}{\partial \theta}=-2 v_{r} \frac{G M}{r^{2}}
$$

We did not find a general solution of the system (B.D)-(B.5), but have found a self-similar solution of this system in the form

$$
v_{r}=-\sqrt{2 G M / r} f(\theta), \quad v_{\theta}=\sqrt{2 G M / r} g(\theta), \quad \rho=\rho(\theta), \quad \mathbf{B}=a \rho \mathbf{v}, a=\text { const. }
$$

The last relation (B.]) is connected with a coincidence of the magnetic field and the streamlines directions, follows from comparison of Eqs. (B.3) and (B.4). Substituting two first relations from (B.7) into Eq.(B.6) we find that it is satisfied when

$$
f^{2}+g^{2}=1
$$

It is convenient, using (B.]),(B.8), to transfer from $(f, g, B, v)$ to new variables $(z, y)$

$$
f=\cos z, \quad g=\sin z \quad y=\frac{a^{2}}{4 \pi} \rho=\frac{B^{2}}{4 \pi \rho v^{2}} .
$$

Here $y$ is equal to the ratio of the magnetic and kinetic energies. For $y$ and $z$, we have two equations of the first order

$$
\begin{aligned}
& \frac{d y}{d \theta}=y(1-y) \frac{\cot z-\cot \theta}{\sin ^{2} z-y} \cdot \sin ^{2} z \\
& \frac{d z}{d \theta}=\frac{1}{2}-\frac{\sin z \cos z(\cot z-\cot \theta)}{\sin z-y} y
\end{aligned}
$$

A solution for zero magnetic field is $y=0, z=\theta / 2$. Using Eq. (B.3), instead of the third relation (B.7), we obtain the solution in the form [四]

$$
v_{r}=-\sqrt{2 G M / r} \cos (\theta / 2), \quad v_{\theta}=\sqrt{2 G M / r} \sin (\theta / 2), \quad \rho(r, \theta)=\rho_{0} \Phi\left(\frac{r}{R} \sin ^{2} \frac{\theta}{2}\right) \tan \theta / 2,
$$

where $\Phi$ is an arbitrary function. This solution describes axially symmetric streamline around a gravitational center of particles flow with a possible angular momentum at infinity [14]]. We present here a solution with the spherically-symmetric distribution of matter at infinity. Deviations from the spherical symmetry around a BH appear only due to the magnetic field. It was shown in [ [, , []], that the only physically relevant solution exist at $y=1$, denoting equipartition between magnetic and kinetic energies everywhere. In this case only one equation remains

$$
\frac{d z}{d \theta}=\frac{3}{2}-\tan z \cdot \cot \theta ; \quad \text { with the boundary condition } \quad z(0)=0,
$$


corresponding to the radial motion of the matter on the pole. The numerical solution of Eq. (B.]3]) in the region $0 \leq<\theta \leq \pi / 2$ is obtained in [प]. The density in this solution is constant $\rho=\rho_{0}$, and the velocity $v$ is equal to the Alfven velocity $v_{A}=\frac{B}{\sqrt{4 \pi \rho}}$. The pattern of the streamlines, coinciding with magnetic force lines is shown schematically by dashed lines in Fig. [1], see [ [U]]. Equation ([.]3]) does not change under a mirror transformation $\theta \rightarrow \pi-\theta$ and $z \rightarrow-z$. So, the solution is antisymmetrically extended to the lower hemisphere $\pi / 2<\theta \leq \pi$, with $v_{r}$ having the same sign as at the upper hemisphere (directed to the centre,) and $v_{\theta}$ changes its sign (see Fig.(B)). Therefore the plane $\theta=\pi / 2$ occurs to be singular. In this plane, a quasi-stationary disk is formed, in which matter moves to a $\mathrm{BH}$, penetrating through magnetic field lined due to finite electrical conductivity.

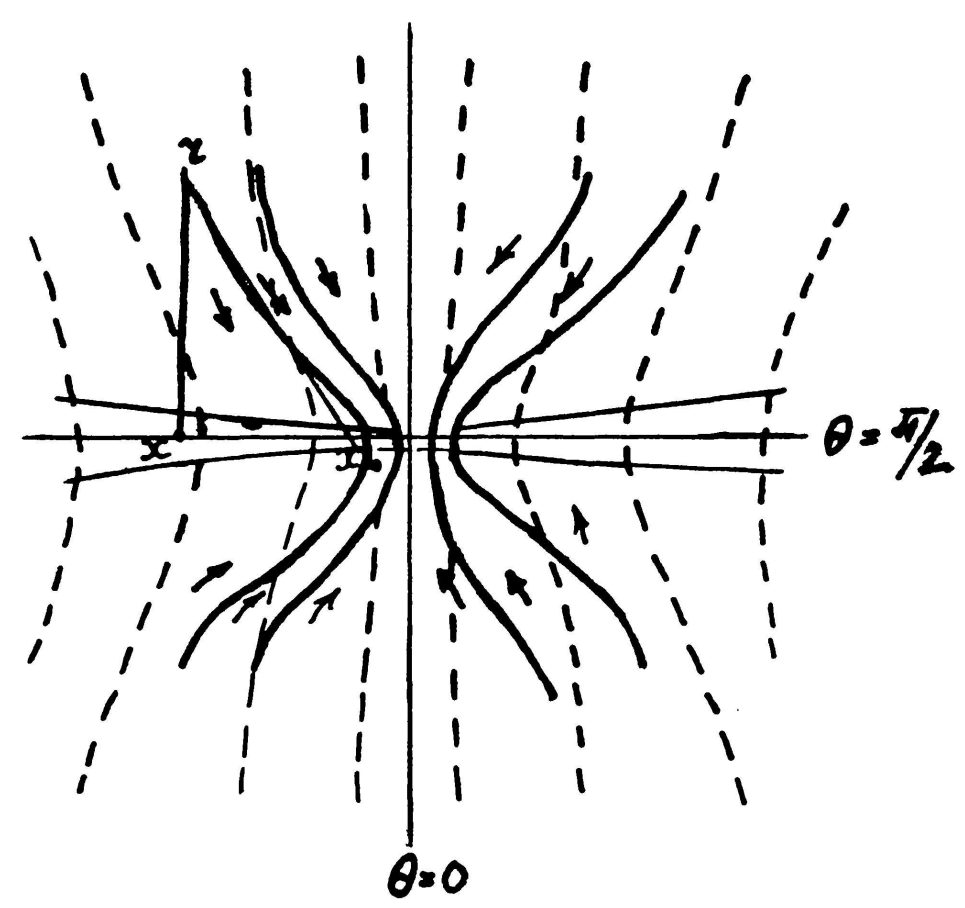

Figure 3: Schematic pattern of magnetic field lines in the matter around a BH for a field which is uniform at infinity, with the inclusion of distortions due to disk currents. The non-perturbed external magnetic field is shown by dashed lines. The solid lines determine the magnetic field lines, influenced by the azimuthal electrical currents in the disk. The arrows indicate a direction of a gas flux velocity, with account of perturbations from the disk, from [[]].

\section{Accretion disk around BH with large scale magnetic field (non-rotating disk)}

If the characteristic scale of non-uniformity of magnetic field is much larger, that the accretion radius $r_{a}$, the flow looses its spherically symmetry,

$$
r_{\mathrm{a}}=\frac{G M}{v_{s}^{2}}, \quad \text { where } v_{s} \text { is the sound velocity in the gas. }
$$

For a uniform magnetic field the accretion symmetry is cylindrical. If a BH is at rest, a stationary pattern of magnetic lines is established, the gas flows along them and forms a disk in the plane 


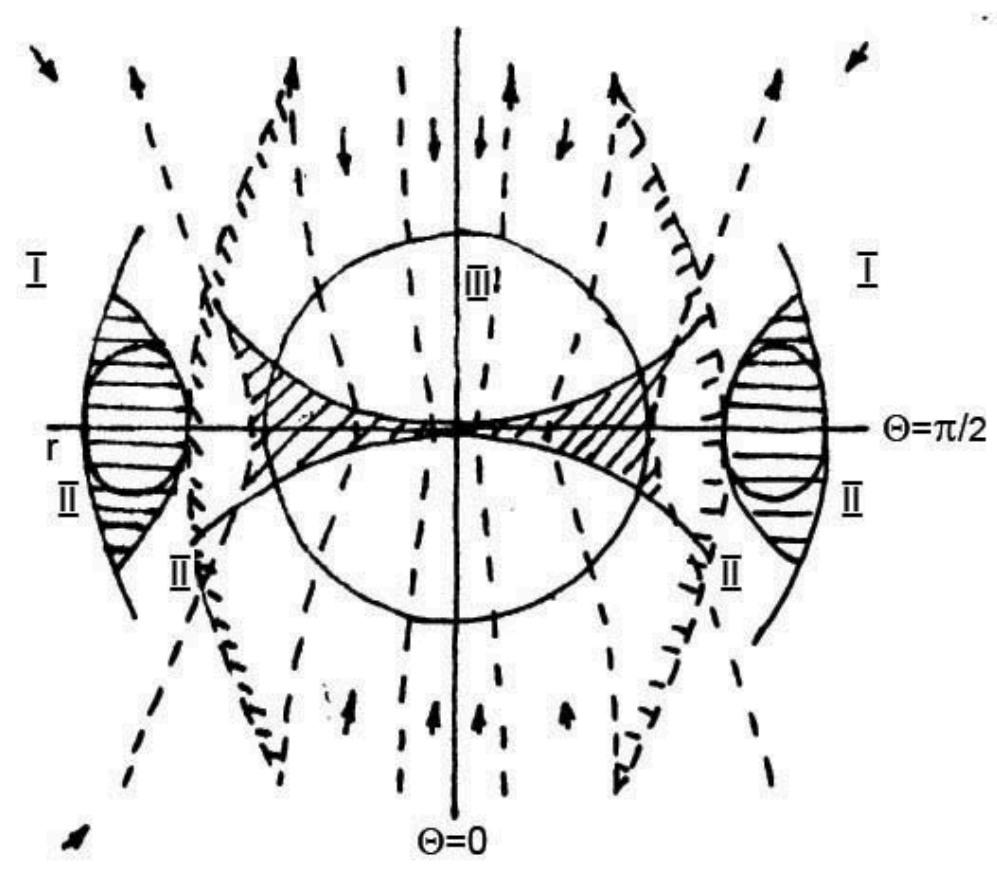

Figure 4: Three zones around a BH during accretion of a gas with a frozen magnetic field, homogeneous at infinity. (I) A zone of the stationary hydrodynamic flow with a non-stationary magnetic field. (II) A zone of the stationary flow in which the transition from the subsonic to the supersonic flow takes place. (Ill) A zone of the stationary supersonic flow. Dashed line marks a place where the velocity reaches the critical sound value. At the plane perpendicular to the magnetic field direction at infinity, thin disk forms around a $\mathrm{BH}$. In zone II the disk becomes thicker and merges with the surrounding flow. Arrows in the direction of the flow velocity have opposite signs in the lower and upper parts, the magnetic field has the same direction there. The ring-like stagnation zone formed in the subsonic region of the stationary flow and situated around the symmetry plane is shown by the horizontal strokes. The sizes of two inner zones are slowly increasing with time, see [四].

of symmetry. A qualitative picture of the flow is shown in Fig. B. At a finite conductivity in the disk a gas infiltrates through force lines of magnetic field towards a $\mathrm{BH}$. The formation process, the structure of a disk supported by a magnetic field and its radiation had been considered in [प]]. Let us consider an equilibrium of a non-rotating disk with a balance between magnetic forces and gravity:

$$
\frac{G M \Sigma}{r^{2}}=\frac{1}{c} B_{\theta} I_{\varphi} \approx \frac{2 \pi}{c^{2}} I_{\varphi}^{2} .
$$

Here, $\Sigma=2 h \rho$ is the surface density, $\rho$ is the average density of the gas, $I_{\varphi}$ is a circular electrical current surface density. We have roughly [B]

$$
B_{\theta} \approx B_{r} \approx \frac{2 \pi}{c} I_{\varphi}
$$

Equilibrium along $z$ axis is supported by a balance between the vertical pressure gradient, and gravity

$$
\frac{d P}{d z}=-\frac{\rho G M}{r^{2}} \frac{z}{r}, \quad h \approx\left(\frac{r^{3}}{G M} \frac{P}{\rho}\right)^{1 / 2} .
$$


The disk heating due to extraction of the gravitational energy, is related to a slow motion of the disk into a $\mathrm{BH}$, and due to additional heating by matter falling to the disk along magnetic field lines, at an almost free-fall velocity. Finally we obtain an expression for the energy flux from unit disk surface in the form

$$
F=\frac{G M \dot{M}}{4 \pi r^{3}}\left[1+\frac{1}{2}\left(\frac{r}{R}\right)^{3 / 2}\right] .
$$

From the mass conservation law we obtain an expression for the radial velocity of the disk matter $v_{r d}$ in the form

$$
v_{r d}=-\frac{\dot{M}}{2 \pi r \Sigma}\left[1-\left(\frac{r}{R}\right)^{3 / 2}\right] .
$$

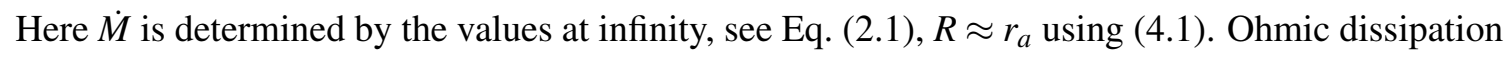
takes place in the disk, leading to gas motion through the magnetic field. The surface electrical current density $I_{\varphi}$ is determined by an equation

$$
\frac{G M \dot{M}}{4 \pi r^{3}}\left[1-\left(\frac{r}{R}\right)^{3 / 2}\right]=\frac{I_{\varphi}^{2}}{4 \pi \sigma},
$$

where $\sigma$ is a conductivity. If the disk is opaque in vertical direction to the radiation, the energy is carried to its surface by a radiative heat conductivity, so that we have approximately

$$
a c T^{4}=\kappa \Sigma F
$$

where $T(r)$ is the mean disk temperature. For an optically transparent disk we have

$$
2 F=\Sigma\left(\varepsilon_{f f}+\varepsilon_{B}\right) .
$$

This relation includes plasma bremsstrahlung $\varepsilon_{f f}$ and magneto-bremsstrahlung $\varepsilon_{B}$. The equation of state in optically thin region is determined by a gas pressure $P_{g}=\rho \mathscr{R} T, \mathscr{R}$ is the gas constant. In optically thick region the radiation pressure should be added $P_{\text {rad }}=a T^{4} / 3$. The input into opacity $k=k_{e}+k_{k}$ comes from the electron scattering $k_{e}=$ and krammers opacity $f f+f b$

$$
\begin{gathered}
\kappa_{f f}+\kappa_{b f} \approx 2 \times 10^{24} \rho T^{-7 / 2} \quad \text { at } k T \ll m_{e} c^{2} ; \quad \kappa_{f f}=5 \times 10^{18} \frac{\rho}{T^{3}} \text { at } k T \gg m_{e} c^{2}, \\
\kappa_{e s}=0.19\left(1+X_{H}\right) .
\end{gathered}
$$

Here $X_{H}$ is the hydrogen mass fraction in accreting matter. The coefficient of the turbulent electrical conductivity is approximated by an expression [ []

$$
\sigma \approx \sigma_{t u r b} \approx \frac{c^{2}}{\tilde{\alpha} 4 \pi h \sqrt{P / \rho}}, \quad \tilde{\alpha}=0.1-0.01
$$

Equations (4.2)-(4.9) with known functions $P, \kappa, \sigma, \varepsilon_{f f}$ and $\varepsilon_{B}$ on the average disk temperature $T$ and density $\rho$, determine a structure of a non-rotating disk with magnetic field around a $\mathrm{BH}$ [四]. The efficiency of the magnetically supported disk, which was called in [12] as magnetically arrested disk (MAD), is very high, about 0.5 , because the gravitational energy always has time to 

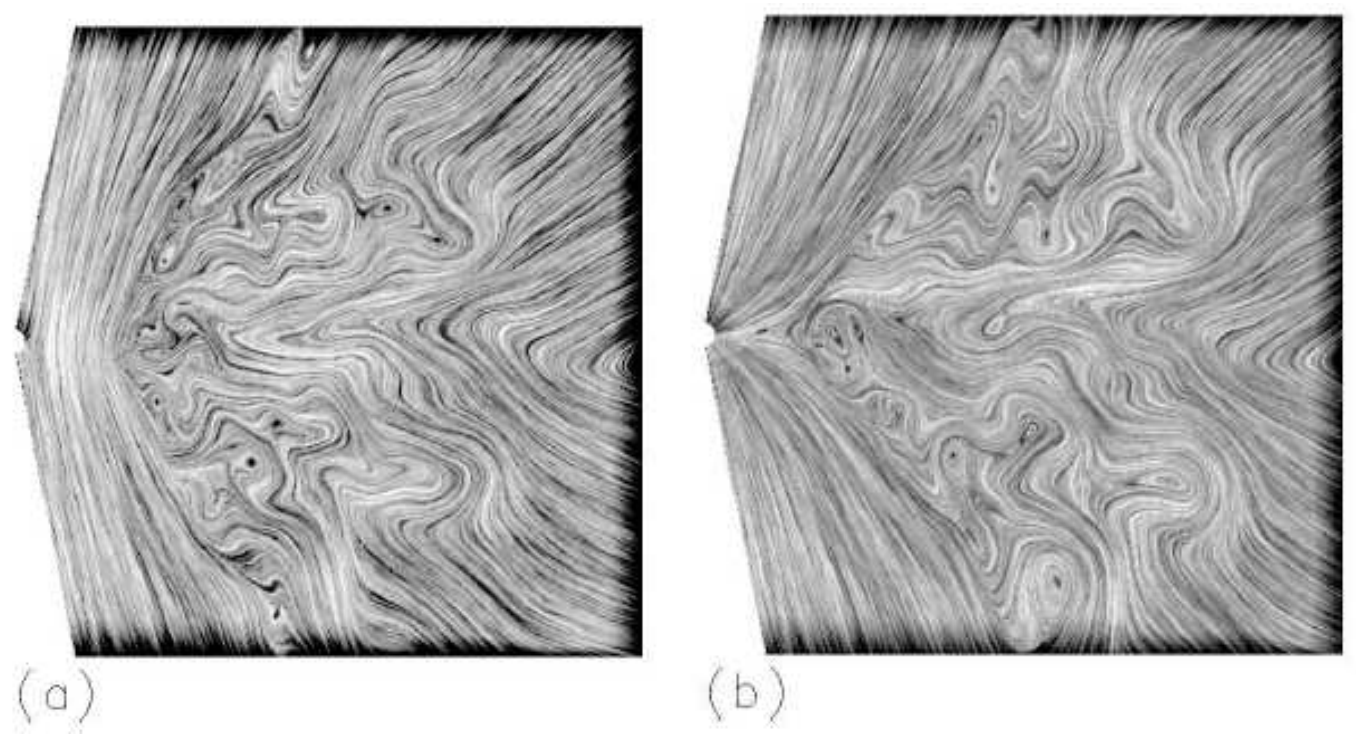

Figure 5: Snapshot of magnetic lines in model with $\beta_{\text {ing }}=100$, at two subsequent moments. The BH is located on the left, and small open circle corresponds to the inner boundary around the black hole at $R_{i n}=2 R_{g}$. The axis of rotation is in the vertical direction. The domain in the figure has a radial size $100 R_{g}$ along the equatorial plane and represents a fraction of the full computational domain with $R_{\text {out }}=220 R_{g}$. The poloidal field lines lying in the meridional plane are shown. The accretion disk transports the vertical magnetic flux inward, which is accumulated in the vicinity of a BH. Small-scale magnetic loops are the result of turbulent motions in the disk and disk corona. (a) Period of accretion, in which most of the accumulated magnetic flux is outside the black hole horizon. (b) Accretion period, in which all the accumulated flux goes through the horizon, [ए]].

convert into heat during stationary disk accretion. For the inner radius of $\sim 1.5 r_{g}$, the luminosity of the stationary turbulent disk is written as

$$
L \approx \frac{G M \dot{M}}{r_{g}}=\frac{1}{2} \dot{M} c^{2}=5 \cdot 10^{31}\left(\frac{M}{M_{\odot}}\right)^{2}\left(\frac{\rho_{\infty}}{10^{-24} \mathrm{~g} \mathrm{~cm}^{-3}}\right) \times\left(\frac{T_{\infty}}{10^{4} \mathrm{~K}}\right)^{-3 / 2} \mathrm{erg} \mathrm{s}^{-1},
$$

and magnetic field distribution over the turbulent disk is written as [प]

$$
B=10^{9} \mathrm{Gs}\left(\frac{M_{\odot}}{M}\right)^{1 / 2}\left(\frac{r_{g}}{r}\right)^{3} \alpha^{-1 / 2}, \quad r_{g}=\frac{2 G M}{c^{2}} .
$$

The general picture of the MAD accretion of a non-rotating gas onto a $\mathrm{BH}$ is given in the Fig.⿴囗十.

\section{Numerical simulations of the magnetically arrested disk}

The authors [ㅁ] have found a lucky name for the old model of magnetically supported disk, stressing the high energetic efficiency of such accretion flow. Numerical simulations of this model, where rotation was included, have shown a rapid angular momentum transfer outward from the central regions of the accretion disk, so that matter is falling onto a black hole being almost nonrotating. One of the first such simulation [ए]] confirmed in general the formation of MAD model. The results of this simulation are presented in Fig.5. Two-temperature, magnetically arrested disc 
simulations of the jet from the supermassive black hole in M87 have been done in [Q], using full general relativistic description. The results, which are qualitatively similar to [ए]], are shown in Fig.6. In both simulation the mildly relativistic jet was formed, and energy extraction from the rotating black hole was important for its formation.

The self-similar solution, and a structure of the disk, supported by magnetic field are obtained analytically for the nonrotating matter accretion. This solution may be applied only for inner parts of the accretion disk, where angular momentum is carried away by magnetic stresses, according to simulation in [Q, [U] $]$. The jet formation, obtained in simulations happens together with a high-efficiency transformation into the radiation flux, in presence of a rotating $\mathrm{BH}$, with additional energy supply from its rotational energy. Alternative accretion model with low radiative efficiency, and main energy flux in the form of the disk wind outflow (ADIOS model) was discussed in [8].

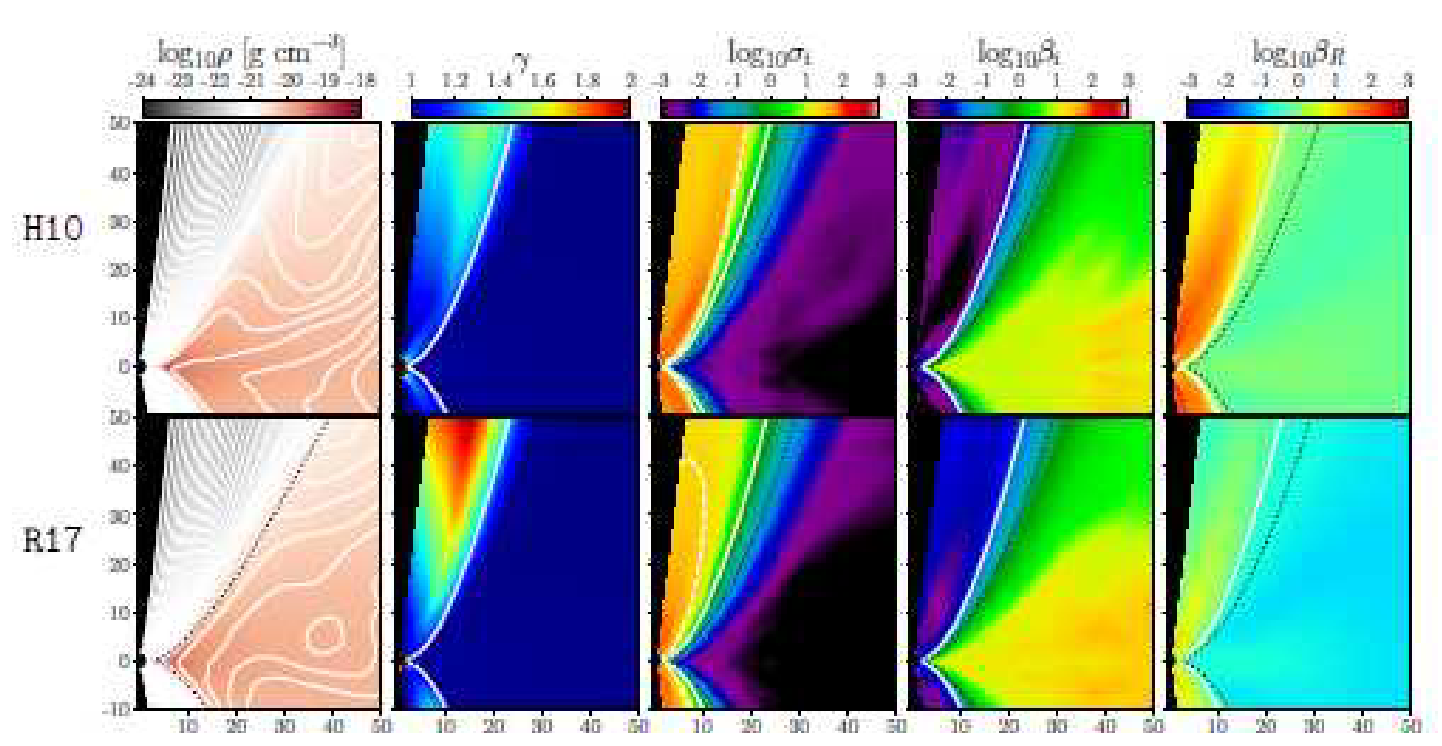

Figure 6: Additional time- and azimuth-averaged properties of the two simulations. From left to right, the quantities displayed are the density $\rho$ in $\mathrm{g} \mathrm{cm}^{-3}$, the bulk Lorentz factor $\gamma$, the plasma magnetization $\sigma_{i}$, the ratio of ion thermal pressure to magnetic pressure $\beta_{i}$, and the ratio of radiation pressure to thermal pressure $\beta_{R}$. In the first column, white contours show the poloidal magnetic field in the averaged data. In the remaining columns, the solid white contour denotes the $\sigma_{i}=1$ surface. The dashed black contour shows the $\mathrm{Be}=0.05$ surface defining the jet boundary. The dashed white contour in the third panel shows the $\sigma_{i}=25$ surface; this is the maximum $\sigma_{i}$ included in the radiative transfer, from [Q] $]$.

Note at the end, that:

*) Quasars and AGN contain supermassive black holes.

**) More than ten $X$ - ray binaries in the Galaxy have stellar mass black holes. These binaries with high mass optical components show variabilities at different time scales, and jet formation, similar to quasars, and are called as microquasars.

***) Jets are observed in objects with black holes, which, most probably, are collimated ejections from magnetized accretion disks [प]]. 


\section{Acknowledgements}

This work was partially supported by RFFI grants No.17-02-00760, 18-02-00619, and RAS Program of basic research 12 "Problems of Origin and Evolution of the Universe".

\section{References}

[1] Bisnovatyi-Kogan, G.S. Stellar Physics: 2: Stellar Evolution and Stability. Astronomy and Astrophysics Library, Springer-Verlag Berlin Heidelberg, 2011.

[2] Bisnovatyi-Kogan G.S., Universe 5, 146 (2019).

[3] Bisnovatyi-Kogan, G.S. and Blinnikov, S.I. Astrophys. Space Sci. 19, 119-144 (1972).

[4] Bisnovatyi-Kogan, G.S. and Friedmann, A.M. Astron. Zh. 46, 721 (1969).

[5] Bisnovatyi-Kogan, G.S. and Lovelace R.V.E. Astrophys. J. 486, L43-L46 (1997).

[6] Bisnovatyi-Kogan G.S., \& Ruzmaikin A.A., Astrophys. and Space Sci. 28, 45 (1974).

[7] Bisnovatyi-Kogan G.S., \& Ruzmaikin A.A., Astrophys. and Space Sci. 42, 401 (1976).

[8] Blandford R.D. and Begelman M.C. Mon. Not. R. Astron. Soc. 303, L1-L5 (1999).

[9] Chael, A.; Narayan, R.; Johnson, M.D. eprint arXiv:1810.01983 2018.

[10] Igumenshchev, I.V. Astrophys. J. 677, 317-326 (2008).

[11] Lovelace, R.V.E. Nature, 262, 649-652 (1976).

[12] Narayan, R.; Igumenshchev, I.V.; Abramowicz, M.A. Publ. Astron. Soc. of Japan, 55, L69-L72 ( 2003).

[13] Shvartsman, V.F. Halos around "Black Holes". Astron. Zh. 48, 479 (1971).

[14] Spiegel, E.E. The Gas Dynamics of Accretion. in H. J. Habing (ed.), Interstellar Gas Dynamics. D. Reidel Publishing Company, Dordrecht-Holland, 201, 1970. 